\title{
COMPACT SOURCE OF ELECTRON BEAM WITH ENERGY OF 200 KEV AND AVERAGE POWER OF 2 KW
}

\author{
V.L. Auslender, V.E. Balakin, A.V. Bulatov, A.A. Bryazgin, I.I. Glazkov, I.V. Kazarezov, \\ E.N. Kokin, A.M. Molokoedov, G.S. Krainov, G.I. Kuznetsov, A.A.Tuvik \\ Budker INP, Novosibirsk, Russia
}

\section{Abstract}

The paper describes a compact electron beam source ELVI-200/2 with average electron energy of $200 \mathrm{keV}$. The source operates with pulse power up to $2 \mathrm{MW}$ under average power not higher than $2 \mathrm{~kW}$, pulse duration $0.2 \div$ $2 \mathrm{mks}$, and repetition rate up to $5 \mathrm{kHz}$. The electron beam is extracted through aluminium - beryllium alloy foil. The pulse duration and repetition rate can be changed from control desk. High-voltage generator for the source with output voltage up to $220 \mathrm{kV}$ is realized using the voltagedoubling circuit which consists of 30 sections. The insulation type - gas, $\mathrm{SF}_{6}$ under pressure of $8 \mathrm{~atm}$. The cooling of the foil supporting tubes is provided by a water - alcohol mixture from an independent source.

\section{INTRODUCTION}

Rapid development of radiation technology on the base of electron beams in the last quarter of XXth century had promoted creation at BINP a series of electron accelerators, both continuous (type ELV [1]), and pulse (ELIT [2], ILU [3]). In a number of cases the pulse electron beams proved to be more preferred in comparison with continuous ones [4]. Sometimes there is a need of compact sources of electron beam. A compact pulse electron source to be created for the radiation technology is described below.

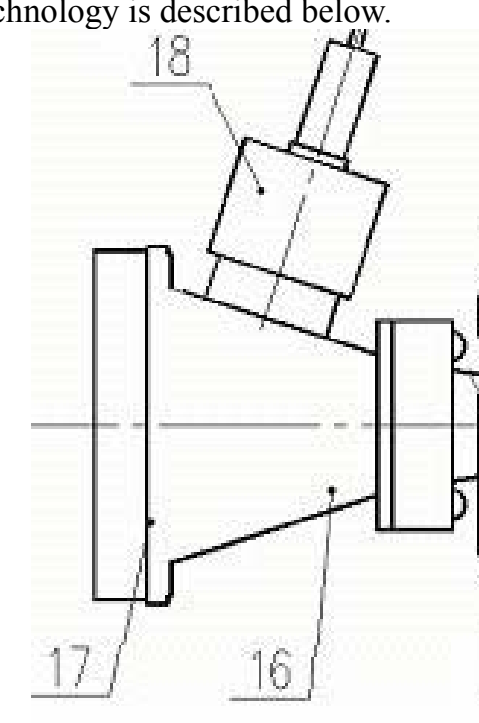

\section{TECHNICAL CHARACTERISTICS}

Average electron energy in the beam, $\mathrm{keV} \quad 200$

Average beam power, $\mathrm{kW}$

Pulse beam current, A

Beam window dimensions, $\mathrm{mm} \quad 180 \times 60$

Energy spread in the beam, \% $\quad+10 /-30$

The source can function in two extreme modes:

\begin{tabular}{|l|c|l|}
\hline \multicolumn{1}{|c|}{ Mode } & 1 & 2 \\
\hline Pulse duration, mks & 2 & 0.2 \\
\hline Pulse repetition rate, $\mathrm{Hz}$ & 500 & 5000 \\
\hline
\end{tabular}

Sort of insulation - gas, $\mathrm{SF}_{6}$ under pressure of $8 \mathrm{~atm}$.

Foil cooling - by air, cooling of supporting tubes - by water - alcohol mixture from an independent source.

\section{DESIGN OF ELECTRON BEAM SOURCE}

The design of high-voltage generator (HVG) and electron beam source is shown in Fig. 1. High voltage generator converts the alternating voltage with frequency of $1 \mathrm{kHz}$ into a direct voltage using the full-wave rectifier and the single-phase transformer. The feeding voltage

Figure 1: Electron accelerator. 1 - primary winding; 2 - input of primary winding; 3 - section of a secondary winding; 4 - magnetic circuit; 5 - high-voltage electrode; 6 - accelerating tube; 7 - electron gun power supply and control unit; 8 - isolating transformer for power transfer; 9 - loop couplers; 10 - cathode-grid unit; 11 - focusing electrode; 12 - insulator; 13 - potential divider; 14 - a water-cooled jacket; 15 - high pressure vessel; 16 - scanning horn; 17 - beam window; 18 - ion pump. 
is supplied to the primary winding of the transformer 1 through input 2. The HVG rectifier comprises 30 rectifying sections connected in series. The primary winding and a column formed by rectifying sections are installed coaxially on the magnetic circuit core 4 . The magnetic circuit is made twisted from the electrical steel (type 3421), thickness of $0.15 \mathrm{~mm}$. It is impregnated with epoxy compound. The primary winding is made coneshaped to reduce leakage inductance. The magnetic circuit and the primary winding are covered by the shield to protect the primary winding in case of possible breakdowns of high-voltage insulation. The high-voltage part of a secondary winding is ended by a high-voltage electrode 5 with the radius of curvature providing rather low working gradients of electric field tension. The highvoltage electrode is connected to the accelerating tube (AT) 6 through the electron gun power supply and control unit (GCU) 7. The GCU is fed through the isolating transformer 8 . The control voltage pulse is transmitted through the loop coupler 9 or fiber bundle. The cathodegrid unit 10 fed from GCU and focusing electrodes 11 are installed inside the accelerating tube. The focusing electrodes 11 form the electron beam and protect the ceramic insulators 12 from the charged particles. The potential divider 13 is placed on the outer side of the accelerating tube. It provides the uniform voltage distribution along the accelerating tube and takes away the charge of the electrons that hits the focusing electrodes 11 . The protective spark gaps are connected to the rings of the divider protect accelerating tube from energy liberation at breakdowns. The water cooling jacket 14 removes heat from the transformer and other components. All units of source are placed inside high pressure vessel 15 .

The electron beam has an elongated form at the output of the accelerator due to focusing by quadruple lens. This beam is directed into the scanning horn 16 having beam window 17 made of aluminium-berillium alloy $\mathrm{AB}$. The foil is cooled by air. It is supported from its vacuum side by the tubes (cooled by independent water - alcohol mixture cooling system) to reduce the operating temperature in it. The chosen width of tubes provides transparency for beam passage not worse than $90 \%$. The pumping of the accelerating tube and scanning horn is carried out by ion pump 18. The Figures 2-3 show HVG and sections of secondary winding combined with rectifiers.

\section{DESCRIPTION OF ELECTRON BEAM SOURCE FUNCTIONING}

The block diagram of the electron beam source is presented in Fig. 4. The voltage from the mains ( 3 phases, $220 / 380 \mathrm{~V}, 50 / 60 \mathrm{~Hz}$ ) goes to the rectifier I1. The rectified voltage feeds the AC converter that generates the $\mathrm{AC}$ voltage having frequency of $1 \mathrm{kHz}$ and amplitude up to $200 \mathrm{~V}$. This voltage feeds the primary winding of the high voltage transformer. Each from 30 section of the secondary winding is supplied with its own rectifier made as voltage-doubling circuit. The outputs of the rectifiers are connected in series. The sections are reinforced with the stainless steel rings on the outer and inner sides to avoid the destruction effects during the discharges. The outputs of the rectifier are connected to these rings. The output high voltage is measured with the voltage divider installed in parallel to the rectifier lower section (U2) since the rectifier voltage is directly correlated to the lower section voltage. The signal occurred at the full voltage breakdown is taken from the capacitance divider installed in parallel to the lower section. In case of breakdown in high voltage insulation, there are generated the signals de-energizing the power inverter and prohibiting GCU operation.

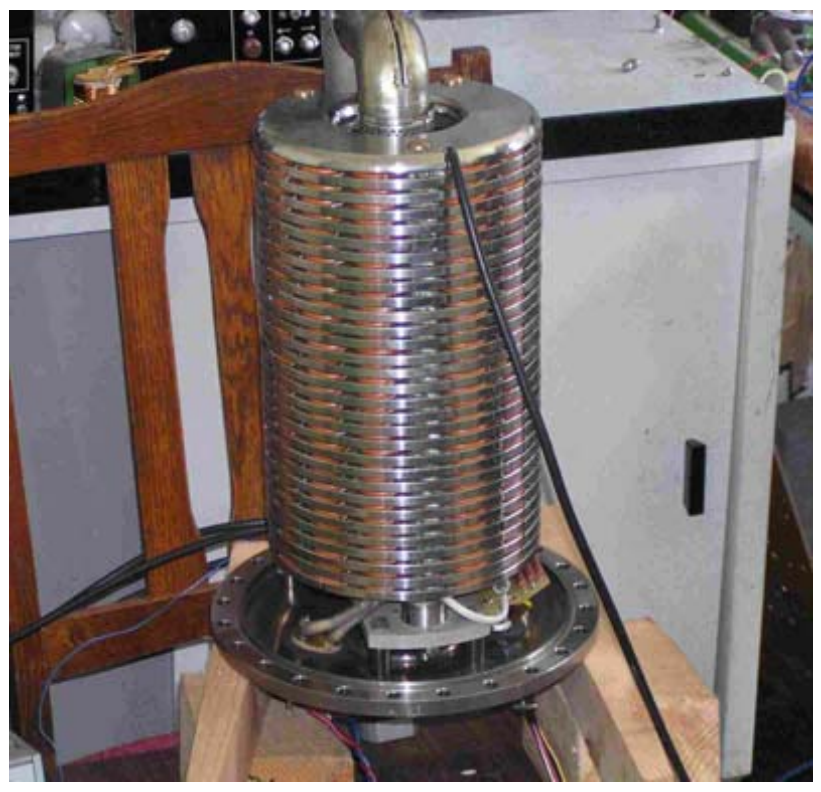

Fig. 2: High voltage generator assembly

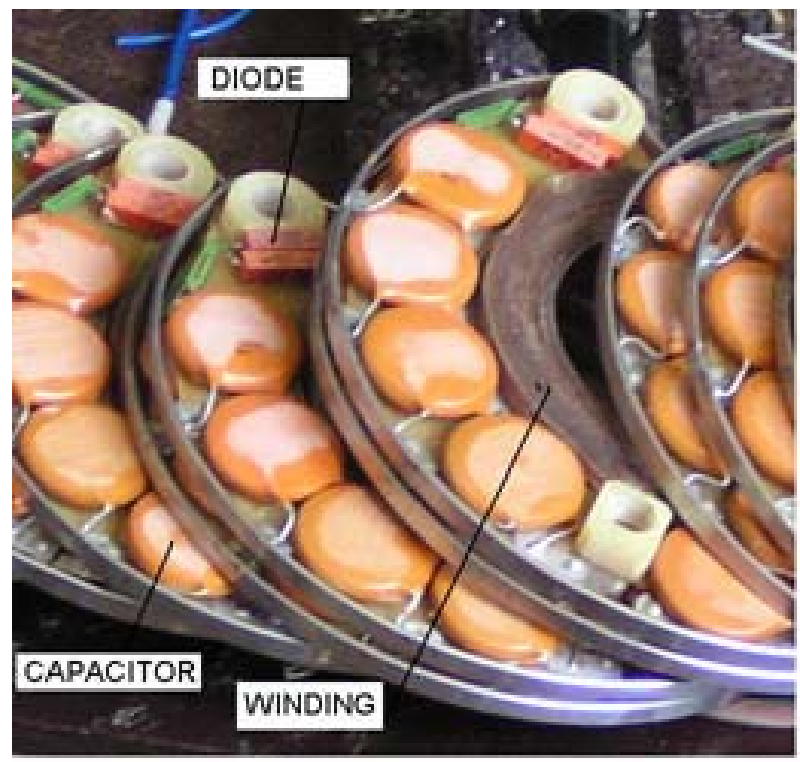

Figure 3: Sections of secondary winding combined with rectifiers. 


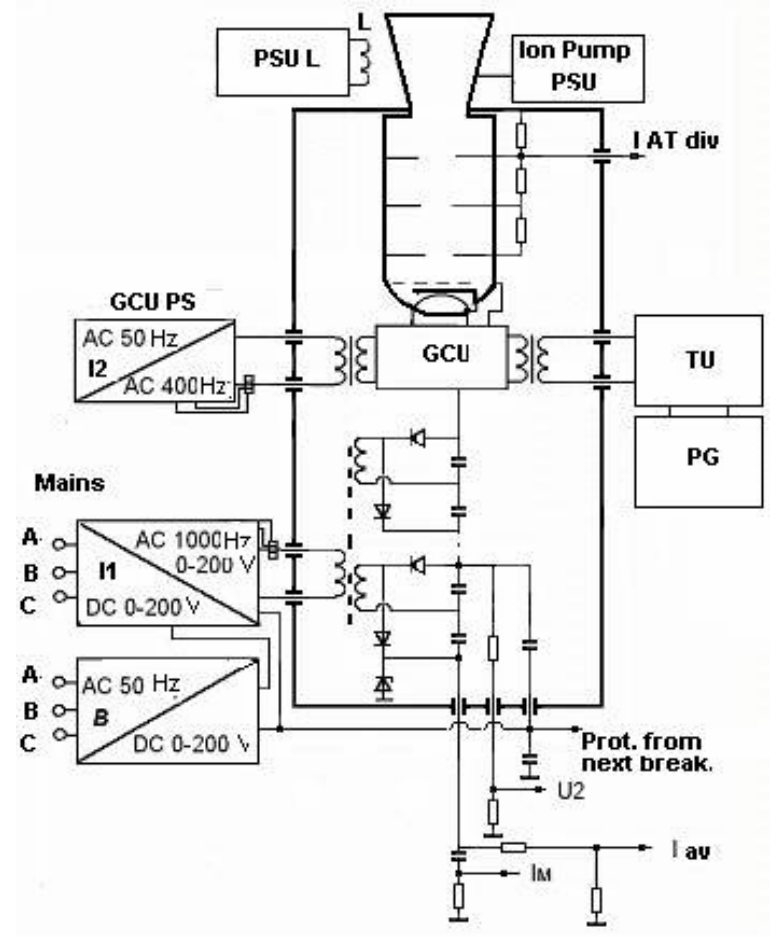

Figure 4: Block diagram of the electron beam source.

The power supply to GCU is applied from the 50/400 $\mathrm{Hz}$ inverter I2 (GCUPS) through the insulating transformer, and control signals - through the coupling loops or fiber bundle from the pulse generator (PG) and trigger unit (TU). Trigger pulses are applied to GCU under conditions that power is applied both to the rectifier and GCU and the ion pump current does not exceed 100 mkA, L-lens is switched on, with the presence of cooling and operation of blocking sets. The power supply units of the lens (PSUL) and ion pump (Ion Pump PSU) provide the feeding of the lens and ion pump. Ion pump current is applied to the control unit and signal unit circuits. If the current value exceeds the allowable one, the control unit cuts the power supply.

\section{TEST RESULTS}

The electron beam source was repeatedly successfully tested on voltage up to $220 \mathrm{kV}$, current up to $10 \mathrm{~A}$, pulse duration $0.2 \div 2 \mathrm{mks}$, average power in beam up to $2 \mathrm{~kW}$ and test duration over the range one hour. The system of next breakdown protection operated safely.

\section{CONCLUSION}

Thus there has been created the compact pulse electron beam source with energy of $200 \mathrm{keV}$. The source operates with pulse power up to $2 \mathrm{MW}$ under average power $2 \mathrm{~kW}$. It can be used both in industry and for science investigations.

\section{REFERENCES}

[1] Accelerators of ELV type: status, development, applications. - Yu. Golubenko, M.E. Veis, N.K. Kuksanov, a.o./ - Novosibirsk, 1997. - 27 p. (Preprint of the BINP SB of Rus.; BINP 97-7)

[2] High voltage pulse electron accelerators INP (Novosibirsk) for industrial and experimental purposes/ S.B. Wasserman, I.V. Kazarezov, V.F. Kucenko, a.o. - In: Proceeding of IV All union conference on particle charge accelerators. Moscow, 1974. Moscow, 1977, v. 1, p. 285-287.

[3] V.L. Auslender ILU-type electron accelerators for industrial technologies Nuclear Instruments \& methods in Physical research, section B, 89,1994, p. 46-48.

[4] W.C. Bradbury Physical and chemical effects of ionizing radiation on cellulosic materials. - In: Intern. Conf. on sterilization by ionizing radiation, Vienna, 1974, Montereal, 1974, p. 387-402. 